\title{
Particle Acceleration in the Cygnus Superbubble
}

\section{Binita Hona ${ }^{a, *}$ on behalf of the HAWC Collaboration}

(a complete list of authors can be found at the end of the proceedings)

${ }^{a}$ University of Utah

Salt Lake City, UT, USA

E-mail: binita.hona@utah.edu

The Cygnus Cocoon is the first gamma-ray superbubble powered by a massive stellar association, the OB2 association. It was postulated that the combined effects of the stellar winds of all the massive O-type stars of the OB2 association can accelerate the cosmic rays to PeV energy in the Cocoon. The conclusive proof of acceleration to PeV energy in the Cocoon will identify the stellar association as a PeV cosmic-ray accelerator, known as PeVatron. However, the Cocoon has been previously studied only up to $10 \mathrm{TeV}$. In this contribution, using 1343 days of High Altitude Water Cherenkov (HAWC) observatory data, we present the morphological and spectral study of the Cocoon above $1 \mathrm{TeV}$ to beyond $100 \mathrm{TeV}$. The analysis at higher $\mathrm{TeV}$ energies reveals a softer spectrum compared to the $\mathrm{GeV}$ gamma-ray observation. This result suggests that the accelerator's efficiency decreases around hundreds of $\mathrm{TeV}$, or after being accelerated, the highest-energy protons escape the region. The study above $10 \mathrm{TeV}$ presented here demonstrates how $\mathrm{CR}$ accelerators operate in these extreme energies and how particle transport impacts high-energy emission.

$37^{\text {th }}$ International Cosmic Ray Conference (ICRC 2021)

July 12th-23rd, 2021

Online - Berlin, Germany

\footnotetext{
*Presenter
} 


\section{Introduction}

Stellar superbubbles, low gas density cavities formed by the interaction between the supersonic winds of massive type $\mathrm{O}$ stars are Galactic cosmic ray (CR) factories and are capable of accelerating cosmic rays to high energies [12]. They might be the source of the CRs accelerated up to the "knee" of the CR spectrum or PeV acceleration in our Galaxy [7]. The Cygnus region in our Galaxy contains massive molecular gas clouds, luminous HII regions and one of the closest giant starforming complexes, called OB2 association. Hence, it is among the best places where gamma rays would be produced in association with the stellar clusters. The first stellar superbubble was discovered by Fermi-Large Area Telescope (LAT) at GeV energies in the Cygnus region [5]. The Fermi-LAT detected an extended region of gamma-ray emission between the two astrophysical objects, the OB2 association and the Gamma Cygni Supernova remnant (SNR). The Fermi-LAT study concluded that this extended emission is due to a single gamma-ray source rather than the multiple components [5]. The infrared emission surrounds the extended gamma-ray emission as in a stellar cocoon, hence the superbubble is also known as Fermi-LAT Cocoon. The morphology of the Cocoon emission is described by a symmetric Gaussian with a width of $2^{\circ}$ and the spectral energy distribution is described by a power law spectrum with a hard index of -2 [5]. The hard index implies that the CRs have not traveled too far from their source. Since there is no evidence of a shockwave from gamma Cygni SNR toward the direction of the superbubble, the SNR is unlikely to be the source powering the superbubble [5]. However, the collective effects of stellar winds from the massive stars in the OB2 association can explain presence of accelerated CRs in the Cocoon [5].

This $\mathrm{GeV}$ emission is co-located with a large extended region of emission detected by the Milagro observatory at TeV energy, MGRO J2031+41 [1]. The previously unidentified MGRO J2031+4157 could be a TeV counterpart of the Fermi-LAT Cocoon [5]. The emission at TeV energies has also been confirmed by the ARGO-YBJ observatory. The ARGO J2031+41, a possible counterpart of the Fermi-LAT cocoon, is co-located with the GeV Cocoon and is described by a 2D Gaussian with a Gaussian width of $1.8^{\circ}$ [11]. The ARGO-YBJ collaboration reported the spectral measurements for this $\mathrm{TeV}$ Cocoon up to $10 \mathrm{TeV}$ [11]. If we want to understand whether the stellar superbubble is capable of CR acceleration up to PeV energies, we need to study the gamma-ray emission beyond $10 \mathrm{TeV}$. In this study, using the data collected by the High Altitude Water Cherenkov (HAWC) observatory, we report the detection and spectral measurements of the $\mathrm{TeV}$ superbubble beyond $10 \mathrm{TeV}$ [4].

The wide field of view HAWC TeV gamma-ray observatory is located at Sierra Negra, Puebla, Mexico at $4100 \mathrm{~m}$. The main array of HAWC consists of 300 water Cherenkov detectors, each with 4 PMTs, and is sensitive to $300 \mathrm{GeV}$ to beyond $100 \mathrm{TeV}$ gamma rays. In the second HAWC catalog, 2HWC J2031+415 was detected near the Fermi-LAT Cocoon [3]. For the study presented here, using 1343 days of HAWC data, a detailed analysis of the 2HWC J2031+415 region was performed to understand the $\mathrm{TeV}$ Cocoon component above $1 \mathrm{TeV}$.

\section{Analysis and Description of the Region}

The modelling of the region was done with the help of the publicly available multi-mission maximum likelihood (3ML) and HAWC accelerated Likelihood (HAL) software. The region of 
the interest (ROI) used for the study is a $6^{\circ}$ disk centered at the Fermi-LAT Cocoon location (RA $=307.17$, Dec $=41.17)^{\circ}$ [4]. The nearby bright source 3HWC J2019+367 was excluded from this ROI by using a $2^{\circ}$ disk mask. The single-source and multi-source models were tested for the ROI. The test statistics (TS) of a model fit is given by likelihood ratio test [16], TS $=2 \ln \frac{L_{\text {(source) }}}{L_{\text {(no-source) }}}$, where $L_{\text {(source) }}$ is the maximum likelihood of a model including a source and $L_{\text {(no-source) }}$ is the maximum likelihood of a model without the source in question. According to Wilks' theorem, the TS follows a $\chi^{2}$ distribution with the degree of freedom equal to the number of the free parameters in case of the nested models [17]. The model with the best TS was chosen to describe the ROI. This model includes three sources as listed in Table 1. Based on the significance distribution of the residual map, this three source model is the best representation of the TeV gamma-ray emission in the ROI [4].

\begin{tabular}{cccc}
\hline Source & ${\text { (RA, Dec })^{\circ}}^{\text {TS }}$ & Morphology \\
\hline & & & \\
HAWC J2030+409 & $(307.65,40.93)$ & 195.2 & Gaussian width of $2.1^{\circ}$ \\
HAWC J2031+415 & $(307.90,41.51)$ & 298.5 & Gaussian width of $0.27^{\circ}$ \\
2HWC J2020+403 & $(305.27,40.52)$ & 53.7 & Disk radius of $0.63^{\circ}$
\end{tabular}

Table 1: The three sources in the ROI.

In the model used to describe the ROI, 2HWC J2031+415 emission is disentangled into two sources: HAWC J2031+415 and HAWC J2030+409. HAWC J2031+415, a small extended Gaussian emission described by the Gaussian width of $0.27^{\circ}$, is possibly associated with a known pulsar wind nebula (PWN), TeV J2032+4130, which was first detected by HEGRA [6] and has been later reported by other observatories such as MAGIC [8], WHIPPLE [13], and VERITAS [10]. HAWC $\mathrm{J} 2030+409$, a large extended source, is the TeV counterpart of the Cocoon. In addition to these two sources, there is also a third source in the ROI, 2HWC J2020+403, which lies $2.36^{\circ}$ away from the centre of 2HWC J2031+415 location and is associated with the Gamma Cygni SNR [9]. The residual map after subtracting HAWC J2031+415 and 2HWC J2020+403 is shown in Figure 1.

\section{Results: Morphology, Spectrum and Particle Modelling}

HAWC J2030+409 or the HAWC Cocoon co-located with the Fermi-LAT Cocoon is detected with a TS of 195.2 at $\left(\mathrm{RA}=307.65^{\circ} \pm 0.30^{\circ}, \mathrm{Dec}=40.93^{\circ} \pm 0.26^{\circ}\right)$. The emission morphology in HAWC data is described by a Gaussian profile with a width of $2.13^{\circ} \pm 0.15^{\circ}$. The spectral energy distribution is described by a power-law spectrum where the pivot energy is $4.2 \mathrm{TeV}$. The flux normalization is $N_{0}=9.3_{-0.8}^{+0.9}$ (stat.) $\times 10^{-13} \mathrm{~cm}^{-2} \mathrm{~s}^{-1} \mathrm{TeV}^{-1}$ and the spectral index is $\Gamma=-2.64_{-0.05}^{+0.05}$ (stat.) [4]. The energy range for the spectral measurement is $0.75 \mathrm{TeV}$ to $210 \mathrm{TeV}$. The fluxes in 8 energy bins are shown in Figure 1. The flux obtained is compatible with the ARGO YBJ measurement at lower $\mathrm{TeV}$ energies [11] and with an extrapolation from the $\mathrm{GeV}$ measurement $[2,5]$. However, while the $\mathrm{GeV}$ spectrum has a hard spectral index of $\Gamma=-2.1$, a significant softening of the energy spectral density is observed at $\mathrm{TeV}$ range with $\Gamma=-2.6$ in both ARGO-YBJ up to $10 \mathrm{TeV}$ [11], and beyond $100 \mathrm{TeV}$ with HAWC data [4]. 

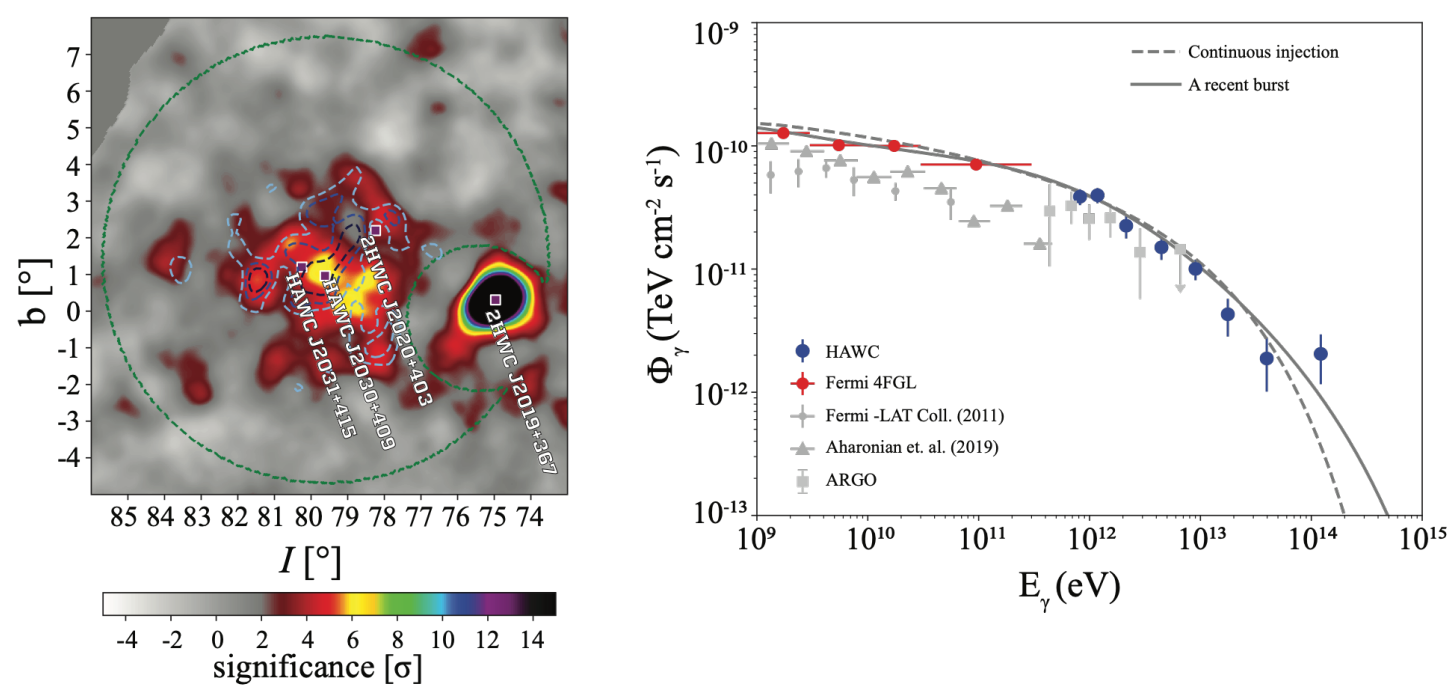

Figure 1: Significance map and the spectral energy distribution of the gamma-ray emission of the HAWC Cocoon [4]. Left: Significance map of the residual emission in the ROI after subtracting HAWC J2031+415 and 2HWC J2020+403. Right: Spectral energy distribution of the Cocoon measured by different $\gamma$-ray instruments, where the blue circles are the spectral measurements for the Cocoon in this study. The errors on the flux points are the $1 \sigma$ statistical errors. The grey solid and dashed lines are $\gamma$-ray spectra derived from the hadronic modeling of the region.

A leptonic model assumed in [4] could not explain the $\mathrm{GeV}$ to $\mathrm{TeV}$ gamma-ray emission, however, two hadronic origin models were provided to explain the gamma-ray spectrum in the Cocoon and the spectral softening of gamma-ray emission at $\mathrm{TeV}$ energies [4]. The grey solid line is the expected gamma-ray distribution assuming a burst model, in which the acceleration process is powered by the recent starburst activities. Assuming a starburst activity less than $0.1 \mathrm{Myr}$ ago and a diffusion length in the Cocoon 100-1000 times shorter than that in the interstellar medium, the CRs are accelerated to PeV energies in the Cocoon [4]. The lower energy CRs remain in the cavity, whereas the highest energy CRs escape the region resulting in a spectral break from the $\mathrm{GeV}$ to the $\mathrm{TeV}$ regime [4]. The grey dotted line is the expected gamma-ray distribution assuming a continuous injection hadronic model, in which the acceleration process is powered by the stellar winds of multiple type $\mathrm{O}$ stars and has continuously injected particles over the OB2 lifetime (1 to 7 Myrs). The spectral softening at TeV energies is due to a cutoff in the injected CR spectrum. Assuming a diffusion length in the Cocoon 100-1000 times shorter than that in the interstellar medium, the cutoff in the injected CR spectrum is obtained at about $300 \mathrm{TeV}$ [4].

\section{Discussion}

The GeV observation by the Fermi-LAT observatory suggests that the OB2 association accelerates CRs and produces gamma-rays with a hard spectrum resulting from an efficient particle acceleration process such as the diffusive shock acceleration. The radial profile of the GeV gammaray emission implies the confinement of freshly accelerated CRs to the center of the Cocoon [4].

The analysis of HAWC data at higher TeV energies reveals new information about the Cocoon. 
In the HAWC band, the gamma-ray spectrum is described by a non-broken power law extending beyond $100 \mathrm{TeV}$ which provides evidence that $\mathrm{PeV}$ cosmic rays were produced in this source. The flux measured by HAWC cannot be explained by inverse Compton scattering of electrons based on the leptonic modelling from [4]. Additionally, the $\mathrm{TeV}$ Cocoon emission region overlaps with the $\mathrm{GeV}$ emission region. Considering the age of the Cocoon, this implies that the diffusion length in the Cocoon is much shorter than that in the interstellar medium. In comparison to $\mathrm{GeV}$ observation, the TeV gamma-ray spectrum softens significantly. This suggests that either the accelerator slows down around hundreds of $\mathrm{TeVs}$ (continuous injection hadronic model), or there is a leakage of the highest-energy protons from the region (burst hadronic model). In this study, the preference of one hadronic model over the other was not conclusive but might be obtained in the future with more statistics at even higher energy.

The observation of the Cocoon above $10 \mathrm{TeV}$ shows how the stellar superbubble operates at extreme energies and provides direct evidence that the superbubble can indeed produce CRs close to the knee region. The detailed study of the superbubble in the future data from the HAWC outriggers array, LHAASO observatory [14], and SWGO observatory [15] will provide a clearer picture of the region.

\section{Acknowledgments}

We acknowledge the support from: the US National Science Foundation (NSF); the US Department of Energy Office of High-Energy Physics; the Laboratory Directed Research and Development (LDRD) program of Los Alamos National Laboratory; Consejo Nacional de Ciencia y Tecnología (CONACyT), México, grants 271051, 232656, 260378, 179588, 254964, 258865, 243290, 132197, A1-S-46288, A1-S-22784, cátedras 873, 1563, 341, 323, Red HAWC, México; DGAPA-UNAM grants IG101320, IN111716-3, IN111419, IA102019, IN110621, IN110521; VIEP-BUAP; PIFI 2012, 2013, PROFOCIE 2014, 2015; the University of Wisconsin Alumni Research Foundation; the Institute of Geophysics, Planetary Physics, and Signatures at Los Alamos National Laboratory; Polish Science Centre grant, DEC-2017/27/B/ST9/02272; Coordinación de la Investigación Científica de la Universidad Michoacana; Royal Society - Newton Advanced Fellowship 180385; Generalitat Valenciana, grant CIDEGENT/2018/034; Chulalongkorn University's CUniverse (CUAASC) grant; Coordinación General Académica e Innovación (CGAI-UdeG), PRODEP-SEP UDG-CA499; Institute of Cosmic Ray Research (ICRR), University of Tokyo, H.F. acknowledges support by NASA under award number 80GSFC21M0002. We also acknowledge the significant contributions over many years of Stefan Westerhoff, Gaurang Yodh and Arnulfo Zepeda Dominguez, all deceased members of the HAWC collaboration. Thanks to Scott Delay, Luciano Díaz and Eduardo Murrieta for technical support. 


\section{References}

[1] A. A. Abdo et al. TeV Gamma-Ray Sources from a Survey of the Galactic Plane with Milagro. Astrophys. J. Lett., 664(2):L91-L94, August 2007.

[2] S. Abdollahi et al. Fermi Large Area Telescope Fourth Source Catalogs. Astrophys. J. Supp., 247(1):33, Mar 2020.

[3] A. U. Abeysekara et al. The 2HWC HAWC Observatory Gamma-Ray Catalog. ApJ, 843(40), 2017.

[4] A. U. Abeysekara et al. HAWC observations of the acceleration of very-high-energy cosmic rays in the Cygnus Cocoon. Nat. Astron., 5:465-471, Mar 2021.

[5] M. Ackermann et al. A cocoon of freshly accelerated cosmic rays detected by fermi in the cygnus superbubble. Science, 334, 2011.

[6] F. Aharonian et al. An unidentified TeV source in the vicinity of Cygnus OB2. Astron. Astrophys., 393:L37-L40, October 2002.

[7] Felix Aharonian, Ruizhi Yang, and Emma de Oña Wilhelmi. Massive stars as major factories of Galactic cosmic rays. Nat. Astron., 3:561-567, Mar 2019.

[8] J. Albert et al. MAGIC Observations of the Unidentified $\gamma$-Ray Source TeV J2032+4130. Astrophys. J. Lett., 675(1):L25, March 2008.

[9] E. Aliu et al. Discovery of TeV Gamma-Ray Emission toward Supernova Remnant SNR G78.2+2.1. ApJ, 770(2):93, June 2013.

[10] E. Aliu et al. Observations of the unidentified gamma-ray source tev j2032+4130 by veritas. Astrophys. J., 783(16), 2014.

[11] B. Bartoli et al. Identification of the TeV Gamma-Ray Source ARGO J2031+4157 with the Cygnus Cocoon. Astrophys. J., 790(2):152, August 2014.

[12] C. J. Cesarsky and T. Montmerle. Gamma-Rays from Active Regions in the Galaxy - the Possible Contribution of Stellar Winds. Space Sci. Rev, 36(2):173-193, October 1983.

[13] M. J. Lang et al. Evidence for $\mathrm{TeV}$ gamma ray emission from $\mathrm{TeV} \mathbf{J} 2032+4130$ in Whipple archival data. Astron. Astrophys., 423:415-419, August 2004.

[14] Andrii Neronov and Dmitri Semikoz. Lhaaso telescope sensitivity to diffuse gamma-ray signals from the galaxy. Phys. Rev. D, 102:043025, Aug 2020.

[15] H. Schoorlemmer. A next-generation ground-based wide field-of-view gamma-ray observatory in the southern hemisphere. In 36th International Cosmic Ray Conference (ICRC2019), volume 36 of $I C R C$, page 785, July 2019.

[16] Giacomo Vianello et al. The Multi-Mission Maximum Likelihood framework (3ML). arXiv e-prints, page arXiv:1507.08343, July 2015. 
[17] S. S. Wilks. The Large-Sample Distribution of the Likelihood Ratio for Testing Composite Hypotheses. Annals Math. Statist., 9(1):60-62, 1938. 


\section{Full Authors List: HAWC Collaboration}

A.U. Abeysekara ${ }^{48}$, A. Albert $^{21}$, R. Alfaro ${ }^{14}$, C. Alvarez $^{41}$, J.D. Álvarez ${ }^{40}$, J.R. Angeles Camacho ${ }^{14}$, J.C. Arteaga-Velázquez ${ }^{40}$, K. P. Arunbabu $^{17}$, D. Avila Rojas ${ }^{14}$, H.A. Ayala Solares ${ }^{28}$, R. Babu ${ }^{25}$, V. Baghmanyan ${ }^{15}$, A.S. Barber ${ }^{48}$, J. Becerra Gonzalez ${ }^{11}$, E. BelmontMoreno $^{14}$, S.Y. BenZvi ${ }^{29}$, D. Berley ${ }^{39}$, C. Brisbois ${ }^{39}$, K.S. Caballero-Mora ${ }^{41}$, T. Capistrán ${ }^{12}$, A. Carramiñana ${ }^{18}$, S. Casanova ${ }^{15}$, O. Chaparro-Amaro $^{3}$, U. Cotti ${ }^{40}$, J. Cotzomi ${ }^{8}$, S. Coutiño de León ${ }^{18}$, E. De la Fuente ${ }^{46}$, C. de León ${ }^{40}$, L. Diaz-Cruz ${ }^{8}$, R. Diaz Hernandez ${ }^{18}$, J.C. Díaz-Vélez ${ }^{46}$, B.L. Dingus ${ }^{21}$, M. Durocher ${ }^{21}$, M.A. DuVernois ${ }^{45}$, R.W. Ellsworth ${ }^{39}$, K. Engel ${ }^{39}$, C. Espinoza ${ }^{14}$, K.L. Fan ${ }^{39}$, K. Fang $^{45}$, M. Fernández Alonso ${ }^{28}$, B. Fick ${ }^{25}$, H. Fleischhack ${ }^{51,11,52}$, J.L. Flores ${ }^{46}$, N.I. Fraija ${ }^{12}$, D. Garcia ${ }^{4}$, J.A. García-González ${ }^{20}$, J. L. García-Luna ${ }^{46}$, G. García-Torales ${ }^{46}$, F. Garfias ${ }^{12}$, G. Giacinti ${ }^{22}$, H. Goksu ${ }^{22}$, M.M. González ${ }^{12}$, J.A. Goodman ${ }^{39}$, J.P. Harding ${ }^{21}$, S. Hernandez ${ }^{14}$, I. Herzog ${ }^{25}$, J. Hinton ${ }^{22}$, B. Hona ${ }^{48}$, D. Huang ${ }^{25}$, F. Hueyotl-Zahuantitla ${ }^{41}$, C.M. Hui ${ }^{23}$, B. Humensky ${ }^{39}$, P. Hüntemeyer ${ }^{25}$, A. Iriarte ${ }^{12}$, A. Jardin-Blicq ${ }^{22,49,50}$, H. Jhee ${ }^{43}$, V. Joshi ${ }^{7}$, D. Kieda ${ }^{48}$, G J. Kunde ${ }^{21}$, S. Kunwar ${ }^{22}$, A. Lara ${ }^{17}$, J. Lee ${ }^{43}$, W.H. Lee ${ }^{12}$, D. Lennarz ${ }^{9}$, H. León Vargas ${ }^{14}$, J. Linnemann ${ }^{24}$, A.L. Longinotti ${ }^{12}$, R. López-Coto ${ }^{19}$, G. Luis-Raya ${ }^{44}$, J. Lundeen ${ }^{24}$, K. Malone ${ }^{21}$, V. Marandon $^{22}$, O. Martinez ${ }^{8}$, I. Martinez-Castellanos ${ }^{39}$, H. Martínez-Huerta ${ }^{38}$, J. Martínez-Castro ${ }^{3}$, J.A.J. Matthews ${ }^{42}$, J. McEnery ${ }^{11}$, P. Miranda-Romagnoli $^{34}$, J.A. Morales-Soto ${ }^{40}$, E. Moreno ${ }^{8}$, M. Mostafá ${ }^{28}$, A. Nayerhoda ${ }^{15}$, L. Nellen ${ }^{13}$, M. Newbold ${ }^{48}$, M.U. Nisa ${ }^{24}$, R. Noriega-Papaqui ${ }^{34}$, L. Olivera-Nieto ${ }^{22}$, N. Omodei ${ }^{32}$, A. Peisker ${ }^{24}$, Y. Pérez Araujo ${ }^{12}$, E.G. Pérez-Pérez ${ }^{44}$, C.D. Rho ${ }^{43}$, C. Rivière ${ }^{39}$, D. Rosa-Gonzalez ${ }^{18}$, E. Ruiz-Velasco ${ }^{22}$, J. Ryan ${ }^{26}$, H. Salazar ${ }^{8}$, F. Salesa Greus ${ }^{15,53}$, A. Sandoval ${ }^{14}$, M. Schneider ${ }^{39}$, H. Schoorlemmer ${ }^{22}$, J. Serna-Franco ${ }^{14}$, G. Sinnis ${ }^{21}$, A.J. Smith ${ }^{39}$, R.W. Springer ${ }^{48}$, P. Surajbali ${ }^{22}$, I. Taboada ${ }^{9}$, M. Tanner ${ }^{28}$, K. Tollefson ${ }^{24}$, I. Torres ${ }^{18}$, R. Torres-Escobedo $^{30}$, R. Turner ${ }^{25}$, F. Ureña-Mena ${ }^{18}$, L. Villaseñor ${ }^{8}$, X. Wang ${ }^{25}$, I.J. Watson ${ }^{43}$, T. Weisgarber ${ }^{45}$, F. Werner ${ }^{22}$, E. Willox ${ }^{39}$, J. Wood ${ }^{23}$, G.B. Yodh ${ }^{35}$, A. Zepeda ${ }^{4}$, H. Zhou ${ }^{30}$

${ }^{1}$ Barnard College, New York, NY, USA, ${ }^{2}$ Department of Chemistry and Physics, California University of Pennsylvania, California, PA, USA, ${ }^{3}$ Centro de Investigación en Computación, Instituto Politécnico Nacional, Ciudad de México, México, ${ }^{4}$ Physics Department, Centro de Investigación y de Estudios Avanzados del IPN, Ciudad de México, México, ${ }^{5}$ Colorado State University, Physics Dept., Fort Collins, CO, USA, ${ }^{6}$ DCI-UDG, Leon, Gto, México, ${ }^{7}$ Erlangen Centre for Astroparticle Physics, Friedrich Alexander Universität, Erlangen, BY, Germany, ${ }^{8}$ Facultad de Ciencias Físico Matemáticas, Benemérita Universidad Autónoma de Puebla, Puebla, México, ${ }^{9}$ School of Physics and Center for Relativistic Astrophysics, Georgia Institute of Technology, Atlanta, GA, USA, ${ }^{10}$ School of Physics Astronomy and Computational Sciences, George Mason University, Fairfax, VA, USA, ${ }^{11}$ NASA Goddard Space Flight Center, Greenbelt, MD, USA, ${ }^{12}$ Instituto de Astronomía, Universidad Nacional Autónoma de México, Ciudad de México, México, ${ }^{13}$ Instituto de Ciencias Nucleares, Universidad Nacional Autónoma de México, Ciudad de México, México, ${ }^{14}$ Instituto de Física, Universidad Nacional Autónoma de México, Ciudad de México, México, ${ }^{15}$ Institute of Nuclear Physics, Polish Academy of Sciences, Krakow, Poland, ${ }^{16}$ Instituto de Física de São Carlos, Universidade de São Paulo, São Carlos, SP, Brasil, ${ }^{17}$ Instituto de Geofísica, Universidad Nacional Autónoma de México, Ciudad de México, México, ${ }^{18}$ Instituto Nacional de Astrofísica, Óptica y Electrónica, Tonantzintla, Puebla, México, ${ }^{19}$ INFN Padova, Padova, Italy, ${ }^{20}$ Tecnologico de Monterrey, Escuela de Ingeniería y Ciencias, Ave. Eugenio Garza Sada 2501, Monterrey, N.L., 64849, México, ${ }^{21}$ Physics Division, Los Alamos National Laboratory, Los Alamos, NM, USA, ${ }^{22}$ Max-Planck Institute for Nuclear Physics, Heidelberg, Germany, ${ }^{23}$ NASA Marshall Space Flight Center, Astrophysics Office, Huntsville, AL, USA, ${ }^{24}$ Department of Physics and Astronomy, Michigan State University, East Lansing, MI, USA, ${ }^{25}$ Department of Physics, Michigan Technological University, Houghton, MI, USA, ${ }^{26}$ Space Science Center, University of New Hampshire, Durham, NH, USA, ${ }^{27}$ The Ohio State University at Lima, Lima, OH, USA, ${ }^{28}$ Department of Physics, Pennsylvania State University, University Park, PA, USA, ${ }^{29}$ Department of Physics and Astronomy, University of Rochester, Rochester, NY, USA, ${ }^{30}$ Tsung-Dao Lee Institute and School of Physics and Astronomy, Shanghai Jiao Tong University, Shanghai, China, ${ }^{31}$ Sungkyunkwan University, Gyeonggi, Rep. of Korea, ${ }^{32}$ Stanford University, Stanford, CA, USA, ${ }^{33}$ Department of Physics and Astronomy, University of Alabama, Tuscaloosa, AL, USA, ${ }^{34}$ Universidad Autónoma del Estado de Hidalgo, Pachuca, Hgo., México, ${ }^{35}$ Department of Physics and Astronomy, University of California, Irvine, Irvine, CA, USA, ${ }^{36}$ Santa Cruz Institute for Particle Physics, University of California, Santa Cruz, Santa Cruz, CA, USA, ${ }^{37}$ Universidad de Costa Rica, San José, Costa Rica, ${ }^{38}$ Department of Physics and Mathematics, Universidad de Monterrey, San Pedro Garza García, N.L., México, ${ }^{39}$ Department of Physics, University of Maryland, College Park, MD, USA, ${ }^{40}$ Instituto de Física y Matemáticas, Universidad Michoacana de San Nicolás de Hidalgo, Morelia, Michoacán, México, ${ }^{41}$ FCFM-MCTP, Universidad Autónoma de Chiapas, Tuxtla Gutiérrez, Chiapas, México, ${ }^{42}$ Department of Physics and Astronomy, University of New Mexico, Albuquerque, NM, USA, ${ }^{43}$ University of Seoul, Seoul, Rep. of Korea, ${ }^{44}$ Universidad Politécnica de Pachuca, Pachuca, Hgo, México, ${ }^{45}$ Department of Physics, University of Wisconsin-Madison, Madison, WI, USA, ${ }^{46}$ CUCEI, CUCEA, Universidad de Guadalajara, Guadalajara, Jalisco, México, ${ }^{47}$ Universität Würzburg, Institute for Theoretical Physics and Astrophysics, Würzburg, Germany, ${ }^{48}$ Department of Physics and Astronomy, University of Utah, Salt Lake City, UT, USA, ${ }^{49}$ Department of Physics, Faculty of Science, Chulalongkorn University, Pathumwan, Bangkok 10330, Thailand, ${ }^{50}$ National Astronomical Research Institute of Thailand (Public Organization), Don Kaeo, MaeRim, Chiang Mai 50180, Thailand, ${ }^{51}$ Department of Physics, Catholic University of America, Washington, DC, USA, ${ }^{52}$ Center for Research and Exploration in Space Science and Technology, NASA/GSFC, Greenbelt, MD, USA, ${ }^{53}$ Instituto de Física Corpuscular, CSIC, Universitat de València, Paterna, Valencia, Spain 